\title{
Two Compasses in the Central Complex of the Locust Brain
}

\author{
Uta Pegel, ${ }^{1}{ }^{\circledR}$ Keram Pfeiffer, ${ }^{2}$ Frederick Zittrell, ${ }^{1}$ Christine Scholtyssek, ${ }^{3}$ and $\odot$ Uwe Homberg ${ }^{1}$ \\ ${ }^{1}$ Animal Physiology, Department of Biology and Center for Mind, Brain and Behavior, Philipps-Universität Marburg, 35032 Marburg, Germany, ${ }^{2}$ Behavioral \\ Physiology and Sociobiology (Zoology II), Biozentrum, University of Würzburg, Am Hubland, 97074 Würzburg, Germany, and ${ }^{3}$ School of Experimental \\ Psychology, University of Bristol, Bristol BS8 1TU, United Kingdom
}

Many migratory insects rely on a celestial compass for spatial orientation. Several features of the daytime sky, all generated by the sun, can be exploited for navigation. Two of these are the position of the sun and the pattern of polarized skylight. Neurons of the central complex (CX), a group of neuropils in the central brain of insects, have been shown to encode sky compass cues. In desert locusts, the CX holds a topographic, compass-like representation of the plane of polarized light (E-vector) presented from dorsal direction. In addition, these neurons also encode the azimuth of an unpolarized light spot, likely representing the sun. Here, we investigate whether, in addition to $E$-vector orientation, the solar azimuth is represented topographically in the CX. We recorded intracellularly from eight types of CX neuron while stimulating animals of either sex with polarized blue light from zenithal direction and an unpolarized green light spot rotating around the animal's head at different elevations. CX neurons did not code for elevation of the unpolarized light spot. However, two types of columnar neuron showed a linear correlation between innervated slice in the CX and azimuth tuning to the unpolarized green light spot, consistent with an internal compass representation of solar azimuth. Columnar outputs of the CX also showed a topographic representation of zenithal $E$-vector orientation, but the two compasses were not linked to each other. Combined stimulation with unpolarized green and polarized blue light suggested that the two compasses interact in a nonlinear way.

Key words: central complex; head direction; insect brain; navigation; polarization vision; sky compass

\section{Significance Statement}

In the brain of the desert locust, neurons sensitive to the plane of celestial polarization are arranged like a compass in the slices of the central complex (CX). These neurons, in addition, code for the horizontal direction of an unpolarized light cue possibly representing the sun. We show here that horizontal directions are, in addition to $E$-vector orientations from the dorsal direction, represented in a compass-like manner across the slices of the CX. However, the two compasses are not linked to each other, but rather seem to interact in a cell-specific, nonlinear way. Our study confirms the role of the CX in signaling heading directions and shows that different cues are used for this task.

\section{Introduction}

Many animals rely on visual cues for navigation. Some of them, including certain insects, exploit global compass cues of the sky to extract heading information and maintain directions during walking and flight (Wehner, 1984; Merlin et al., 2012; Homberg, 2015). Sky compass cues are highly reliable due to their persistent presence during locomotion (Gould, 1998; Frost and Mouritsen,

\footnotetext{
Received April 13, 2018; revised Jan. 10, 2019; accepted Jan. 29, 2019.

Author contributions: U.P., K.P., and U.H. designed research; U.P. performed research; U.P., F.Z., and C.S. contributed unpublished reagents/analytic tools; U.P. analyzed data; U.P. wrote the first draft of the paper; U.P., K.P., F.Z., C.S., and U.H. edited the paper; U.P. and U.H. wrote the paper.

This work was supported by the Deutsche Forschungsgemeinschaft (Grants H0 950/23-1 and HO 950/24-1). We thank Erich Buchner and Christian Wegener (University of Würzburg) for supplying anti-synapsin antibodies and Martina Kern for maintaining locust cultures.

The authors declare no competing financial interests.

Correspondence should be addressed to Uwe Homberg at homberg@biologie.uni-marburg.de.

https://doi.org/10.1523/JNEUROSCI.0940-18.2019

Copyright $\odot 2019$ the authors
}

2006). In addition to direct sunlight, the polarization pattern and the chromatic gradient across the sky, both generated by scattering of sunlight in the atmosphere, provide reference to the position of the sun. In addition to sky compass cues, insects also rely on landmarks and perhaps even map-like mechanisms of orientation, especially in familiar terrain (Collett, 1992; Menzel et al., 2005; Zars, 2009; Wystrach and Graham, 2012). Large landscape features such as coastlines or mountain ranges may also serve as guiding cues for long-distance migrators (Reppert et al., 2016).

Several insect species show orientation behavior dependent on sky compass cues or, under laboratory settings, signals that mimic zenithal sky polarization or solar position. These include honey bees (von Frisch, 1949; Brines and Gould, 1979), desert ants (Wehner and Müller, 2006), dung beetles (Dacke et al., 2003; el Jundi et al., 2014b), fruit flies (Weir and Dickinson, 2012), field crickets (Brunner and Labhart, 1987), and locusts (Mappes and Homberg, 2004). All of these species possess specialized photoreceptors working as $E$-vector analyzers located in the dorsal rim 
A

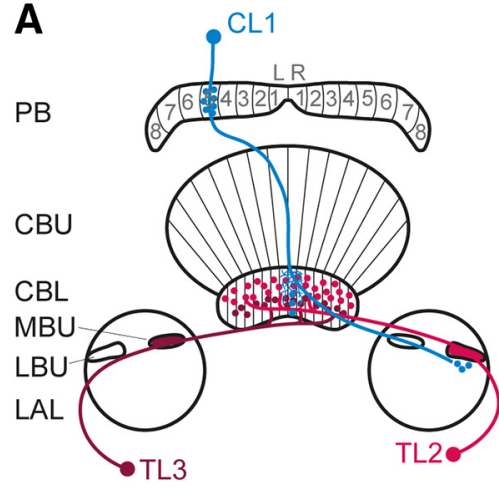

C

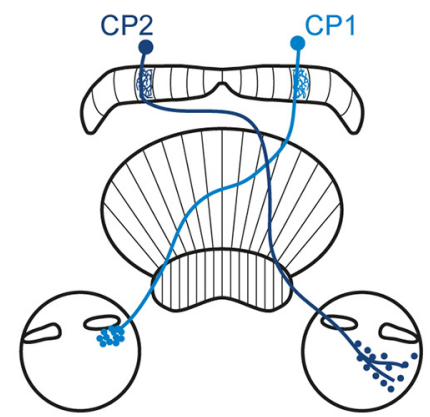

B

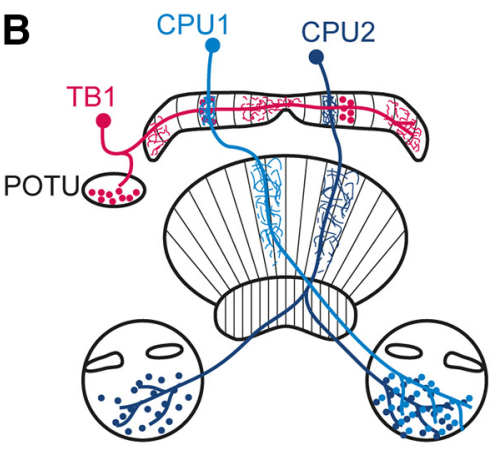

D

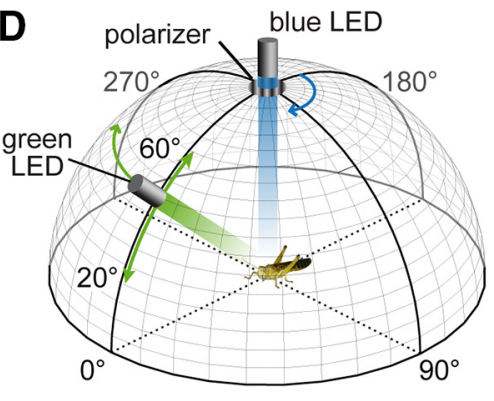

Figure 1. Neuronal cell types and visual stimulation. $\boldsymbol{A}-\boldsymbol{C}$, Schematic illustration of tangential (red) and columnar (blue) neurons of the sky compass network in the locust CX. Vertical lines mark the edges of slices occupied by arborizations of columnar neurons. Slices are termed R1-R8 (right hemisphere) and L1-L8 (left hemisphere). Fine processes illustrate likely dendritic input regions of the neuron; dots represent varicose arborizations und thus likely presynaptic output regions. The filled lateral and medial bulb (LBU, MBU) in $\boldsymbol{A}$ indicate input synapses arranged in microglomerular complexes. LAL, Lateral accessory lobe; POTU, posterior optic tubercle. $\boldsymbol{D}$, Schematic illustration of visual stimulation. The light of a blue LED positioned in the zenith was passed through a rotating polarizer. A green LED appeared to the animal as an unpolarized light spot. It rotated around the animal's head (green horizontal arrow) at elevations ranging from $20^{\circ}$ to $60^{\circ}$ (green vertical arrow).

areas of their compound eyes (Labhart and Meyer, 1999; Schmeling et al., 2014).

Visual pathways from the dorsal rim areas of both compound eyes converge on the central complex (CX), a group of midlinespanning neuropils in the brain (Homberg et al., 2011; Heinze, 2014). The CX consists of the upper and lower division of the central body (CBU and CBL, respectively), the paired noduli $(\mathrm{NO})$, and the protocerebral bridge (PB; Fig. $1 A$ ). In the desert locust, the $\mathrm{CBU}, \mathrm{CBL}$, and $\mathrm{PB}$ are structured in rows of 16 vertical slices. Many CX neurons arborize in adjacent bilateral structures, the lateral accessory lobes, and the medial and lateral bulbs. Several types of CX neuron are involved in the processing of polarized light. These include tangential neurons of the CBL (TL2) serving as input neurons to the $\mathrm{CX}$ and contacting columnar neurons of the CBL (CL1; Fig. 1A). CL1 neurons arborize in distinct slices of the $\mathrm{PB}$, where they might contact tangential neurons (TB1; Fig. 1B). Columnar neurons, termed CPU1, CPU2, $\mathrm{CP} 1$, and $\mathrm{CP} 2$, likely serve as output neurons of the network (Fig. $1 B, C)$.

Functional studies provide strong evidence for a role of the CX in navigational tasks (Ofstad et al., 2011; Varga et al., 2017). In locusts and fruit flies, the CX holds an internal representation of head orientation relative to a visual reference. Whereas in fruit flies head orientation relative to bright landmarks is represented in the ellipsoid body (corresponding to the CBL in other species; Seelig and Jayaraman, 2013) and the PB (Green et al., 2017), a topographic representation of zenithal $E$-vectors is present in the $\mathrm{PB}$ of the locust (Heinze and Homberg, 2007). In dung beetles, monarch butterflies, and locusts, CX neurons code for the orien-

tation of zenithal polarized light as well as for the azimuth of an unpolarized green light spot, likely representing the sun (Heinze and Reppert, 2011; el Jundi et al., 2014a, 2015; Pegel et al., 2018). This raises the question of whether the azimuth of the sun, like the $E$-vector angle, is represented topographically in the slices of the CX. If so, an internal azimuth compass phase shifted by $90^{\circ}$ to the E-vector compass would be expected because this is the angular distance between the zenithal $E$-vector and the solar azimuth in the sky. We show here that several types of columnar neuron in the locust CX represent, not only zenithal E-vectors, but also azimuth angles of unpolarized light cues in a compass-like manner. However, the two compasses are not simply connected by a $90^{\circ}$ relationship.

\section{Materials and Methods}

Animals and preparation. Desert locusts (Schistocerca gregaria) were reared under crowded conditions in a 12/12 h light/dark cycle. Only sexually mature male and female animals at least 1 week after final molt were used for experiments. Animals were mounted onto a metal holder using dental wax with their anterior-posterior body axis oriented vertically (Pfeiffer et al., 2005). Wings and legs were cut off. The head capsule was opened from anterior and fat tissue, tracheal air sacs, and gut were removed to reduce body movements. Mouthparts, leg stumps, and abdomen were immobilized by wax. Muscles close to the brain were cut for further stabilization. A small twisted metal wire was used to support the brain from posterior. The neural sheath covering the central brain was removed to allow access for the electrode. During preparation and intracellular recording the brain was immersed in locust saline (Clements and May, 1974) containing $0.09 \mathrm{~mol} \mathrm{l}^{-1}$ saccharose.

Electrophysiology and visual stimulation. Sharp glass microelectrodes were drawn from borosilicate capillaries (Hilgenberg) using a Flaming/ Brown horizontal puller (P-97; Sutter Instruments). Electrode tips were filled with $4 \%$ Neurobiotin (Vector Laboratories) diluted in $1 \mathrm{~mol}^{-1}$ $\mathrm{KCl}$. Electrode shanks were filled with $1 \mathrm{~mol}^{-1} \mathrm{KCl}$. Neuronal signals were amplified $10 \times$ by a custom-built amplifier (University of Regensburg), visualized by an oscilloscope (DS 1052Eh; Rigol Technologies), digitized by an analog-to-digital converter (CED1401 plus; Cambridge Electronic Design) at a rate of $20 \mathrm{kHz}$, and stored on a PC using Spike2 version 6.02 software (Cambridge Electronic Design). Neuronal responses to polarized and unpolarized light stimuli were studied (Fig. 1D). Polarized light was generated by passing light of a blue LED (Oslon SSL 80, LDCQ7P, $452 \mathrm{~nm}$; Osram Opto Semiconductors, or LXML-PR010500, $447.5 \mathrm{~nm}$, Philips Lumileds) through a polarizer (HNP’B; Polaroid). Both were positioned in the animal's zenith (with respect to its natural head orientation) to stimulate the dorsal part of the eye. The polarized light stimulus covered a visual angle of $32.5^{\circ}$ or $18.6^{\circ}$ and had an intensity of $1.7 \times 10^{13}$ photons $\mathrm{cm}^{-2} \mathrm{~s}^{-1}$. The polarizer was rotated at angular velocities of $40 \%$ or $36 \%$. The unpolarized light spot was generated by light from a green LED (LED535-series, $535 \mathrm{~nm}$, Roithner Lasertechnik, or Oslon SSL 80, LT CP7P, 528 nm; Osram Opto Semiconductors) passing through a diffusor. The unpolarized light stimulus appeared at a visual angle of $16.3^{\circ}$ and had an intensity of $10^{14}$ photons $\mathrm{cm}^{-2} \mathrm{~s}^{-1}$. It was moved around the animal's head at an elevation of $45^{\circ}$ and an angular velocity of $40 \%$ or $36 \%$ s. In experiments testing for elevation-dependent coding the elevation of the light spot could be 
changed to $20^{\circ}, 30^{\circ}, 40^{\circ}, 50^{\circ}$, and $60^{\circ}$ (Fig. $1 D$ ). At the end of the recording, Neurobiotin was injected into the neuron by applying a positive constant current of $\sim 1 \mathrm{nA}$ for 1-4 min.

Histology and image processing. Brains were dissected in locust saline, immersed overnight at $4^{\circ} \mathrm{C}$ in fixative containing $4 \%$ PFA, $0.25 \%$ glutaraldehyde, and $0.2 \%$ saturated picric acid diluted in $0.1 \mathrm{~mol}^{-1}$ PBS. Brains were stored for up to 2 weeks at $4^{\circ} \mathrm{C}$ in sodium phosphate buffer. Subsequently, they were incubated in PBS with $0.3 \%$ Triton X-100 and Cy3-conjugated streptavidin $(1: 1000)$ for $3 \mathrm{~d}$, dehydrated in an ascending ethanol series $(30 \%, 50 \%, 70 \%, 90 \%, 95 \%, 100 \%)$ with 15 min steps, and cleared in a 1:1 mixture of $100 \%$ ethanol and methyl salicylate for 20 min, followed by $1 \mathrm{~h}$ in $100 \%$ methyl salicylate. Finally, brains were embedded in Permount (Fisher Scientific) between two coverslips. For synapsin immunostaining, brains were rehydrated in a decreasing ethanol series $(100 \%, 95 \%, 90 \%, 70 \%, 50 \%, 30 \%)$ in 15 min steps, embedded in albumin/gelatin, fixed overnight in $8 \%$ formaldehyde at $4^{\circ} \mathrm{C}$, and sectioned in $130 \mu \mathrm{m}$ slices using a vibrating-blade microtome (VT 1000S; Leica). Sections were preincubated overnight in PBS with 5\% Triton $\mathrm{X}-100$ and 5\% normal goat serum (NGS) and then incubated for $5 \mathrm{~d}$ at $4^{\circ} \mathrm{C}$ in PBS with $5 \%$ Triton X-100, $1 \%$ NGS, and anti-synapsin antibody (1:50). The monoclonal anti-synapsin antibody was generated in mouse against fusion proteins consisting of glutathione-S-transferase and the Drosophila Syn 1 protein (Klagges et al., 1996) kindly provided by Drs. Erich Buchner and Christian Wegener (University of Würzburg). The antibody labels synapse-rich neuropils in various insect species (Brandt et al., 2005; Kurylas et al., 2008; Held et al., 2016). Following incubation in anti-synapsin, sections were incubated in PBS with 5\% Triton X-100, $1 \%$ NGS, and the secondary antibody (goat anti-mouse) conjugated with Cy5 (1:300) for $3 \mathrm{~d}$ at $4^{\circ} \mathrm{C}$. The sections were finally dehydrated in an increasing ethanol series (as described above), cleared in methyl salicylate (as described above), and mounted in Permount between two coverslips (for a more detailed description of the protocol, see Heinze and Homberg, 2008). Preparations were scanned with a confocal laser scanning microscope (Leica) using a DPSS laser $(561 \mathrm{~nm})$ for detection of Cy3 and a He-Ne laser $(633 \mathrm{~nm})$ for detection of Cy5. Scans were visualized in AMIRA (version 5.4.5; FEI Visualization Sciences Group). Images were processed in Adobe Illustrator CC version 2017.1.0.

Preprocessing of physiological data. Recording traces were visualized using Spike2. Action potentials were detected as events with a thresholdbased mechanism. The data were exported to a mat file. All subsequent analysis was performed using custom functions written in MATLAB version 2017a (The MathWorks).

Experimental design and statistical analysis. For each stimulus presentation, a stimulus-response curve was obtained by calculating the mean spiking activity in $10^{\circ}$ bins. To assess the response of a neuron to a stimulus condition, the stimulus-response curves of all presentations were averaged. At least one clockwise and one counterclockwise rotation of the polarizer/unpolarized light spot were averaged. Responses to clockwise and counterclockwise tests were always pooled in equal numbers to avoid a shift in the preferred calculated angle due to rotation direction. A directed modulation of spike rate by the orientation of the $E$-vector or the azimuth of the unpolarized light spot was determined by an angular-linear correlation analysis (Zar, 1999). The responsiveness of the neuron to a stimulus was indicated by the significance $(\alpha=0.05)$ of the correlation coefficient $\left(r_{\mathrm{al}}\right)$. The coefficient of determination $\left(r_{\mathrm{al}}{ }^{2}\right)$ describes the strength of correlation between $E$-vector orientation or light spot azimuth and the spike rate (for a detailed description, see Pegel et al., 2018). To calculate the preferred $E$-vector or azimuth $\left(\Phi_{\max }\right)$, spike times were transformed into angles by multiplying them with the stimulus rotation velocity. From these angles, the mean vector $\Phi$ was calculated (Batschelet, 1981) and defined as the preferred angle $\left(\Phi_{\max }\right)$. The anti-preferred angle $\left(\Phi_{\min }\right)$ was defined as the angle $180^{\circ}$ to $\Phi_{\max }$ (azimuth tuning) or $90^{\circ}$ to $\Phi_{\max }$ (E-vector tuning). For all analyses of axial data (E-vector stimulation), the angles were doubled (Batschelet, 1981). Background activity was determined by selecting parts of the recording without any stimulation and dividing them into $1 \mathrm{~s}$ bins. In each bin, the spikes were counted. Spike counts were used to calculate the median background activity. The correlation between the location of arborization in the PB and $\Phi_{\max }$ was assessed by a circular-linear correlation analysis as described by Kempter et al. (2012). The slice of PB arborization was used as the linear variable (values ranging from 0 to 15 ) and $\Phi_{\max }$ as the circular variable. A linear regression model was fitted to the circular-linear data by minimizing the circular error between measured and predicted angles (Kempter et al., 2012). The slope of the regression line was used to transform the linear variable into a circular one. Finally, a circular correlation coefficient $(\rho)$ was calculated. No prior assumptions on the data were necessary except for an estimate of the range of reasonable slopes. It was determined as the slope $\alpha$ of the regression line with minimum mean circular distance from the data points within a reasonable range of $\pm 80^{\circ}$ per $\mathrm{PB}$ column (Equation 1 in Kempter et al., 2012). The circular-linear correlation coefficient is an analog to the Pearson's product-moment correlation coefficient for linear-linear data, but with higher validity when analyzing circular-linear associations (Kempter et al., 2012).

For Figures 5 and 6, stimulus-response curves were smoothed. The stimulus-response curve was normalized to the median background activity of the neuron. A smoothing spline was fitted onto the curve using the MATLAB curve-fitting toolbox (smoothing parameter set to $10^{-4}$, 360 array elements). For experiments with different light spot elevations, additional characteristics of the stimulus-response curve were calculated using the smoothed stimulus-response curve: the tuning amplitude, and the tuning width. The amplitude was determined by calculating the difference in normalized spike rate between the peak and the trough of the fit curve. The tuning width was defined as the angular difference between two points on the fit curve at half amplitude.

For experiments with combined stimulation (see Fig. 5), we calculated the relative impact of the green and polarized light stimulation alone on the response to simultaneous stimulation. We followed the assumption that the tuning is constituted as follows:

$$
f(\Phi)=p(\Phi+90) \cdot w_{p}+g(\Phi) \cdot w_{g}
$$

Where $\Phi$ is the stimulus angle in degrees, $f$ is the hypothetic tuning curve to simultaneous stimulation, $p$ is the measured tuning curve to polarized light stimulation, $g$ is the measured tuning curve to green-light-spot stimulation, and $w_{\mathrm{p} \mid \mathrm{g}}$ are the respective weighting factors. Tuning curves were smoothed before calculations. We used an optimization approach to find the weighting factors where the summed absolute difference between the measured response to simultaneous stimulation and $f$ is minimal. To this end, we used the MATLAB built-in function fminbnd to minimize the function $h(w)=\sum_{\Phi} \mid c(\Phi)-(p(\Phi+90) \cdot w$ $\left.+g(\Phi) \cdot w_{g}\right) \mid$, where $c$ is the measured tuning curve to simultaneous stimulation and $w$ is the minimization parameter that was constrained to the interval $(-10,10)$. In each optimization iteration, $w_{\mathrm{g}}$ was calculated by solving $f$ for $w_{\mathrm{g}}$ and inserting the measured tuning curve for the hypothetic one: $w_{g}=\frac{c-p \cdot w}{g}$, where $w$ is the optimization parameter of the current iteration. At $\min (h(w))$, the lowest absolute difference between measured and hypothetic tuning curve $w$ was taken as $w_{\mathrm{p}}$ and $w_{\mathrm{g}}$ was calculated as above.

\section{Results}

Most of the data analyzed here are from recordings presented previously (Pegel et al., 2018). In that study, we analyzed basic response features of the neurons, including their tuning to the plane of polarized light, unpolarized green and UV light spots, response amplitudes, tuning widths, and tuning differences when comparing clockwise and counterclockwise rotations of the stimuli. Here, we investigated whether morphological characteristics of the neurons such as the innervated layer in the CBL and columnar domains in the $\mathrm{PB}, \mathrm{CBU}$, and $\mathrm{CBL}$ correspond to tuning angles of the neurons in a topographic manner. 
A

\section{TL2a}
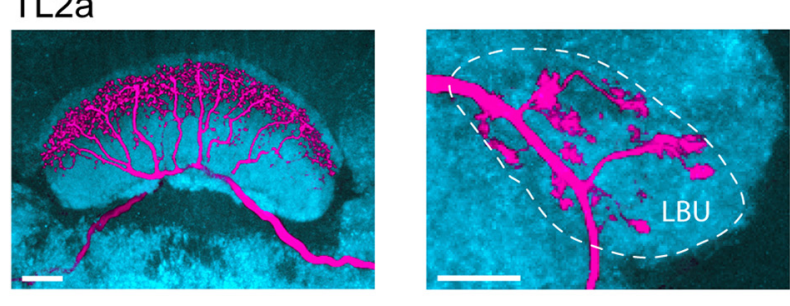

TL2b
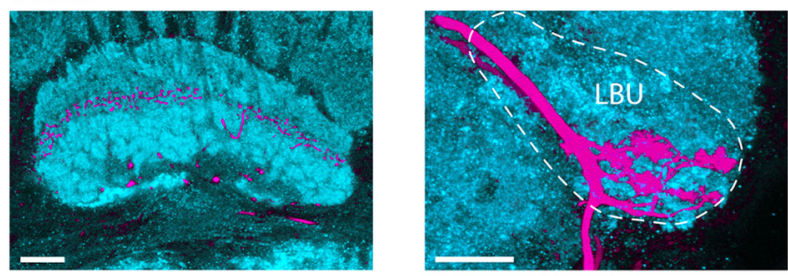

TL3

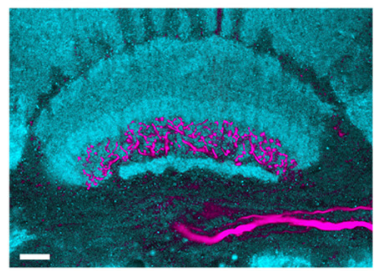

B

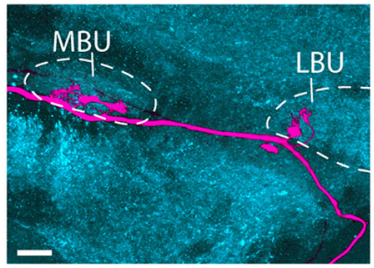

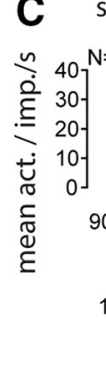

stimulus position $/^{\circ}$

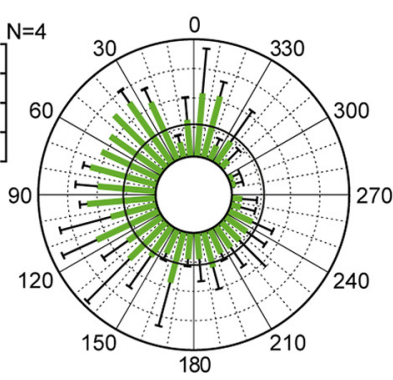

D $\quad \Phi_{\max }{ }^{\circ} 0$
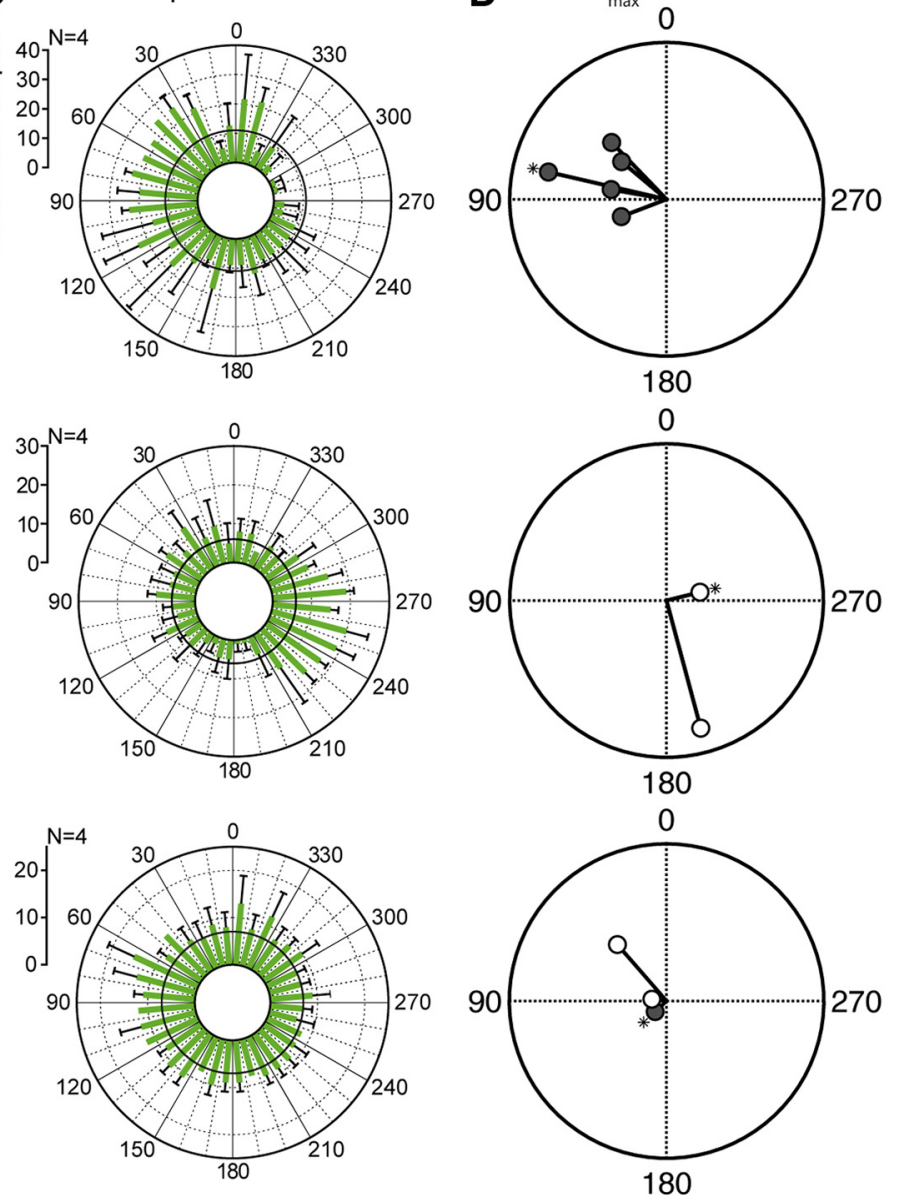

Figure 2. Morphology and physiology of TL neurons. $\boldsymbol{A}, \boldsymbol{B}$, Cy3 stainings of TL neurons (magenta). Neuropils were visualized by synapsin immunostaining (cyan). Scale bars, $20 \mu \mathrm{m}$. $\boldsymbol{A}$, Arborizations of a TL2a neuron (top), a TL2b neuron (middle), and a TL3 neuron (bottom) in the (BL; single optical sections. B, Arborizations of the neurons in the lateral (LBU) or medial bulb (MBU); projections of stacks of several optical sections. C, Circular histograms showing the average response of the TL2a $\left(\Phi_{\max }=76^{\circ}\right), \operatorname{TL} 2 \mathrm{~b}\left(\Phi_{\max }=284^{\circ}\right)$, and TL3 $\left(\Phi_{\max }=133^{\circ}\right)$ neuron presented in $A$ and $B$ to a rotating green light spot (elevation $=45^{\circ}$ ). Green bars indicate mean spiking activity. Error bars indicate SD. Black circles indicate median background activity. $n$, Number of stimulus presentations. D, Population vector averages for the green spot from the recorded TL2a, TL2b, and TL3 neurons (means from two or four green light spot rotations) indicated by their preferred azimuth angle and vector length. Vector length ranges from 0 to unity (outer circle). Open dots indicate a preferred azimuth on the ipsilateral side; filled gray dots indicate a preferred angle on the contralateral side. Asterisks indicate data from the histograms in $\mathbf{C}$.

\section{Tuning angles of TL neurons innervating different layers of the CBL}

To investigate whether azimuthal preference is topographically represented in the layering of the CBL, we compared azimuthal tuning and innervated layer in 10 recorded TL neurons. Six types of tangential neuron termed TL1-TL6 have been distinguished in the CBL of the locust (Müller et al., 1997; Bockhorst and Homberg, 2015). We recorded from seven TL2 and three TL3 neurons, invading different layers of the CBL. Five TL2 neurons invaded layer 2 of the CBL and are termed here TL2a (Fig. 2A). Two TL2 neurons invaded layer 3 and are termed here TL2b (Fig. $2 A$ ). The three TL3 neurons arborized in layers 4 and 5 (Müller et al., 1997). In the lateral bulb, TL2 and TL3 neurons receive signals via microglomerular complexes (Träger et al., 2008). Whereas TL2a neurons innervated microglomeruli in dorsal parts of the lateral bulb, TL2b neurons innervated microglomeruli of more ventral parts (Fig. 2B). In TL3 neurons, arborizations were located in the medial bulb, but one neuron additionally invaded a few microglomeruli at the most mediodorsal tip of the lateral bulb (Fig. 2B). All TL2 neurons responded with excitation at $\Phi_{\max }$ and inhibition at $\Phi_{\min }$. TL3 neurons were generally tuned only weakly to the azimuth of the green spot and either showed exclusively excitation at $\Phi_{\max }$ (Fig. $2 C$ ), inhibition at $\Phi_{\min }$, or both. In all TL2a neurons, the preferred azimuth of the green light spot was on the contralateral side (Fig. $2 C, D$ ). In contrast, in the two TL2b neurons, the preferred azimuth was on the ipsilateral side (Fig. 2C,D). The three TL3 neurons showed mixed responses, with $\Phi_{\max }$ on the ipsilateral side in two neurons and on the contralateral side in the third neuron (Fig. $2 C, D$ ).

\section{Topographic representation of $E$-vector and azimuth angles in the $\mathrm{PB}$}

In the $\mathrm{PB}$, zenithal $E$-vectors are topographically represented, indicating that the $\mathrm{PB}$ acts as an internal sky polarization compass (Heinze and Homberg, 2007). This representation was found for TB1, CPU1, CP1, and CP2 neurons (Heinze and Homberg, 2007), but not for CL1 neurons (Heinze and Homberg, 2009). Here, we investigated whether a topographic representation of azimuth of unpolarized light representing the sun is likewise present across the PB. We recorded from CL1, TB1, CPU1, CP1, and CP2 neurons and from an additional cell type, CPU2 neurons (Fig. $1 A-C$ ). Neurons were tested for $E$-vector coding by presenting polarized light from the zenith and for azimuth coding by presenting an unpolarized green light spot rotating at an 


\section{polarized blue}
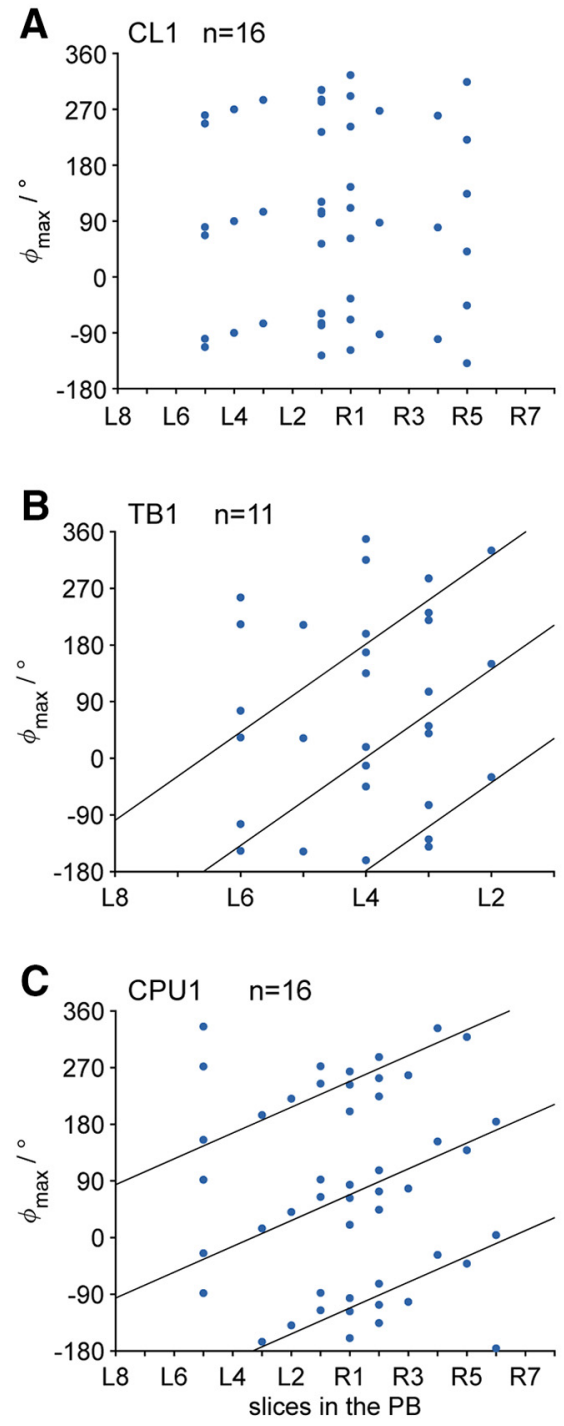

\section{unpolarized green}
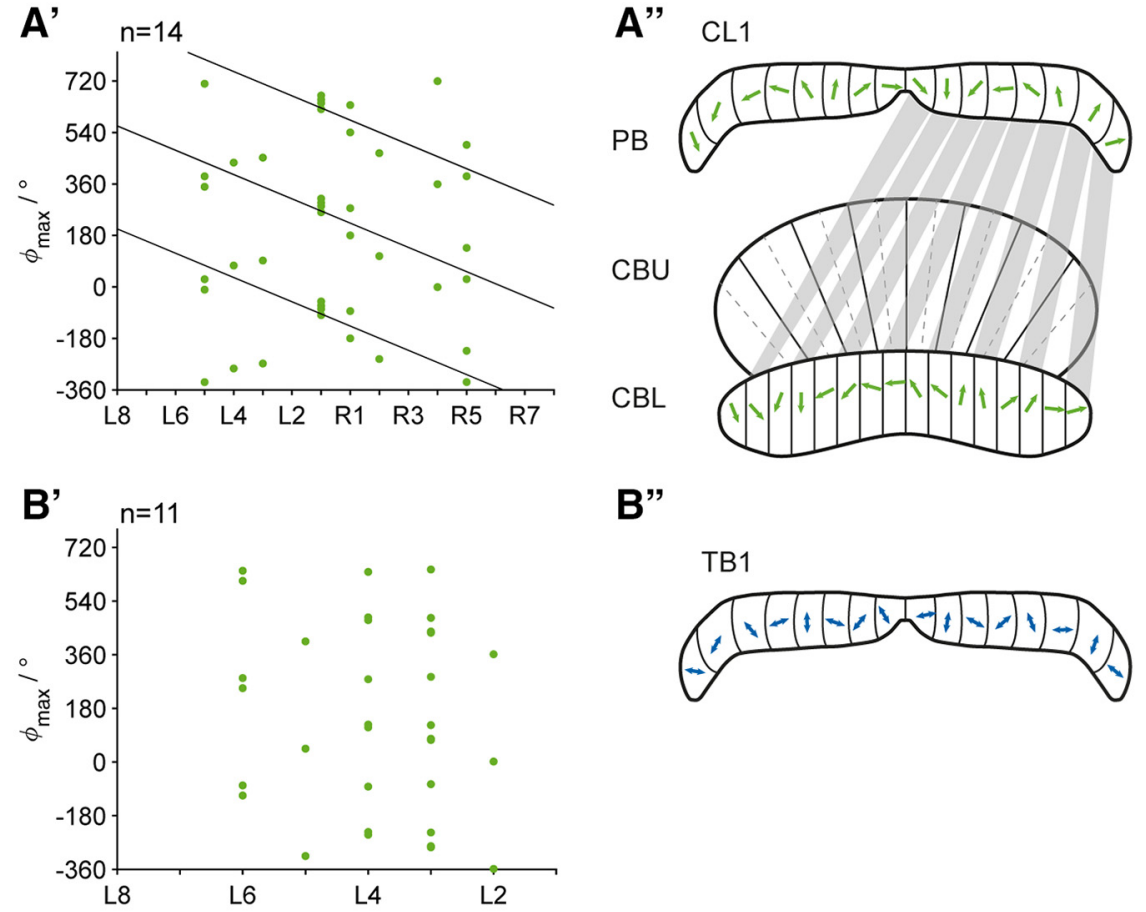

B"

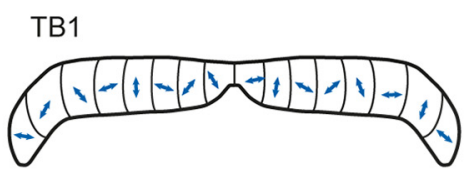

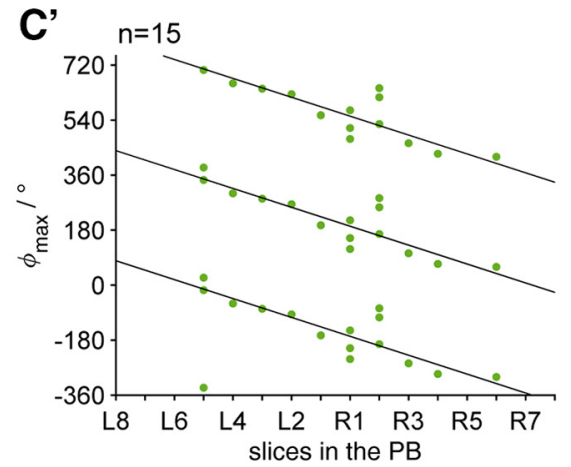

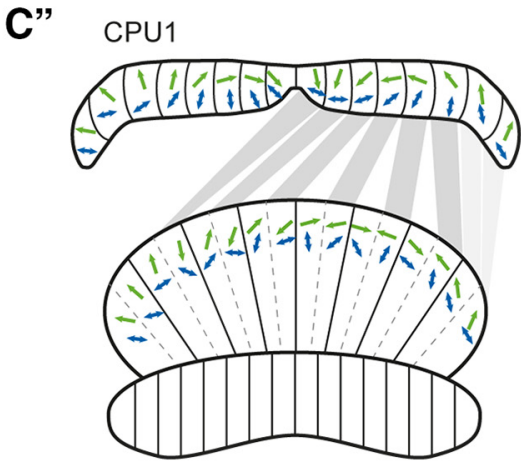

Figure 3. Internal representation of zenithal $E$-vector and azimuth in the $C X$. Preferred $E$-vector angles $\left(\boldsymbol{A}-\boldsymbol{C}\right.$, blue markers) and preferred azimuth angles of the unpolarized light spot $\left(\boldsymbol{A}^{\prime}, \boldsymbol{B}^{\prime}, \boldsymbol{C}^{\prime}\right.$, green markers) are plotted against the slice of arborization of the respective neuron in the PB. Datasets are plotted three times: $\pm 180^{\circ}$ for preferred $E$-vectors and $\pm 360^{\circ}$ for preferred azimuths. Data points are means from two or four stimulus presentations. $n$, Number of recordings. Solid lines show the best fit line only in cases in which $|\rho|>0.25$. Light spot elevation was at $45^{\circ}$. $\boldsymbol{A}^{\prime \prime}, \boldsymbol{B}^{\prime \prime}$, $C^{\prime \prime}$, Mean $E$-vectors (blue double arrows) and mean azimuth angles (green arrows) taken from the circular-linear fits. For neurons arborizing additionally in the (B, the mean preferred angles were transferred from the PB to the CB according to the wiring schemes as shown by Heinze and Homberg (2008). Wiring is indicated by shades of gray for the right brain hemisphere. Light gray indicates unknown and thus hypothetical connections. $\boldsymbol{A}, \boldsymbol{A}^{\prime}$, Preferred angles of $\mathrm{CL} 1$ neurons. Circular-linear regression shows low correlation between innervated slice and preferred angles for polarized blue light $(\rho=0.11)$, but high correlation between innervated slice and unpolarized green light $\left(y=-42.5 x+202.9, \rho=-0.72\right.$ ). $\boldsymbol{B}, \boldsymbol{B}^{\prime}$, Preferred angles of TB1 neurons. Correlation exists for polarized blue light $(y=70.0 x+81.5, \rho=0.60)$ but not for unpolarized green light ( $\rho=-0.04)$. Due to similar location of varicose arborizations of TB1 neurons in the left and right PB hemisphere, only data of the left hemisphere are shown. $\boldsymbol{C}, \boldsymbol{C}^{\prime}$, Preferred angles of (PU1 neurons. Correlation exists for polarized blue light $(y=20.5 x+84.0, \rho=0.33$ ) and unpolarized green light $(y=-30.9 x+80.0, \rho=-0.54)$.

elevation of $45^{\circ}$ around the animals' head. The preferred angles were plotted against the slice of arborization in the PB. Linearcircular regression analysis was performed for CL1, TB1, and CPU1 neurons because only for those cell types sample size was above the critical limit of $n=10$ recordings (Kempter et al., 2012). Because TB1 neurons have varicose arborizations in one $\mathrm{PB}$ slice of each brain hemisphere (separated by seven slices), their preferred angles were plotted only for the left brain hemisphere. In CL1 neurons, the preferred $E$-vector was not correlated with the slice of arborization in the PB (Fig. 3A). These observations are consistent with previous results (Heinze and Homberg, 2009). TB1 neurons showed a correlation between preferred
$E$-vector and slice of arborization (Fig. 3B). The data confirm the results of Heinze and Homberg (2007), who showed a topographic representation of $E$-vectors for TB1 neurons. As in Heinze and Homberg (2007), the fit line had a positive slope. However, it covered an angular range of $490^{\circ}$ across one PB hemisphere, substantially larger than the range of $169.4^{\circ}$ found by Heinze and Homberg (2007). In CPU1 neurons, the preferred $E$-vector angle was highly correlated with the slice of arborization in the PB (Fig. 3C), indicating an internal compass for zenithal $E$-vectors in the PB (Fig. $3 C^{\prime \prime}$ ). The circular-linear fits covered an angular range of $307^{\circ}$ in CPU1 neurons from L8 to R8. The steepness of the fit line was again different from that of Heinze and 
Homberg (2007), who reported a representation of $410^{\circ}$ in CPU1 neurons.

A correlation between the slice of arborization in the PB and the preferred azimuth of the unpolarized light spot was found in CL1 and CPU1 neurons (Fig. $3 A^{\prime}, C^{\prime}$ ). This indicates that, in addition to the $E$-vector of polarized light, the azimuth of the green spot is topographically represented in the $\mathrm{PB}$ (Fig. $3 A^{\prime \prime}, C^{\prime \prime}$ ). However, in contrast to the positive correlation between $E$-vector and $\mathrm{PB}$ slice, the correlation between azimuth and $\mathrm{PB}$ arborization was negative in both cell types. The fits for the preferred azimuth of the unpolarized green spot covered $637.5^{\circ}$ in CL1 neurons and $463.5^{\circ}$ in CPU1 neurons across the PB. Because CPU1 and CL1 neurons arborize, not only in slices of the $\mathrm{PB}$, but also in distinct slices of the central body, we transferred the mean preferred angle (i.e., the $y$-value of the fit line in the center of each slice) from the $\mathrm{PB}$ to the $\mathrm{CB}$ following the wiring scheme of the respective neuron type (Heinze and Homberg, 2008). In CL1 neurons, mean preferred azimuth angles resulted in a representation of $277.1^{\circ}$ across the CBL (Fig. $3 A^{\prime \prime}$ ). CPU1 neurons innervating PB slices L6-R6 arborize in two neighboring slices of the CBU. For the PB slices L8, L7, R8, and R7, however, the wiring scheme is not known because CPU1 neurons arborizing in these slices have never been stained. By extrapolating the logic of connections from the central slices, Heinze and Homberg (2008) suggested that they invade corresponding outermost slices of the CBU. Following that scheme, mean $E$-vector angles across the eight double slices covered a range of $127^{\circ}$ in the $\mathrm{CBU}$ (Fig. $3 C^{\prime \prime}$ ). Interestingly, the mean azimuth angles of CPU1 neurons in double slices of the CBU were almost spatially opponent to each other except for the outermost double slices, which were approximately parallel (Fig. 3C').

Interaction between polarized and unpolarized light stimulus Because the regression lines for preferred $E$-vectors and preferred azimuth angles have different signs, we examined their relationship in more detail. First we investigated whether the angular distance between the tuning to both stimuli ( $E$-vector and green azimuth) and the slice of arborization in the PB were correlated in any way. Across all cell types, distances were independent of the slice of PB arborization (Fig. 4) and varied greatly for a given slice. In CL1 neurons, distances were widely dispersed from $0^{\circ}$ to $180^{\circ}$ (Fig. 4A). In contrast, in most TB1 neurons, distances were smaller than $90^{\circ}$ (Fig. $4 B$ ). In CPU1 neurons, the distances clustered between $45^{\circ}$ and $90^{\circ}$ (Fig. $4 C$ ).

Second, to test for a possible cue preference or linear relationship between the two cues, we presented the unpolarized light spot in combination with the zenithal $E$-vector (Fig. 5). The relative angle between both stimuli was set to $90^{\circ}$, corresponding to the relationship between solar azimuth and zenithal $E$-vector in the sky. We analyzed the responses of two TL2, three CL1, four TB1, six CPU1, two CPU2, and one CP1 neuron. A hypothetical response curve to combined stimulation was calculated to estimate the relative contribution of the single stimuli ( $E$-vector or light spot) to the measured response to combined stimulation. The absolute values and the ratio of weighting factors varied from neuron to neuron and could not be related to cell type, slice of PB innervation, or distance between tuning to the E-vector and green light spot. Weighting factors for azimuth tuning ranged from 0 to 1.7 and, for $E$-vector tuning, from -2 to 1.2. Their ratio ranged from -1.1 to 11.5 . Some neurons showed a strong preference in the combined response for the $E$-vector or the unpolarized light spot, as shown for a CPU1 neuron preferring the $E$-vector (Fig. 5A). Other cells showed a less pronounced prefer-
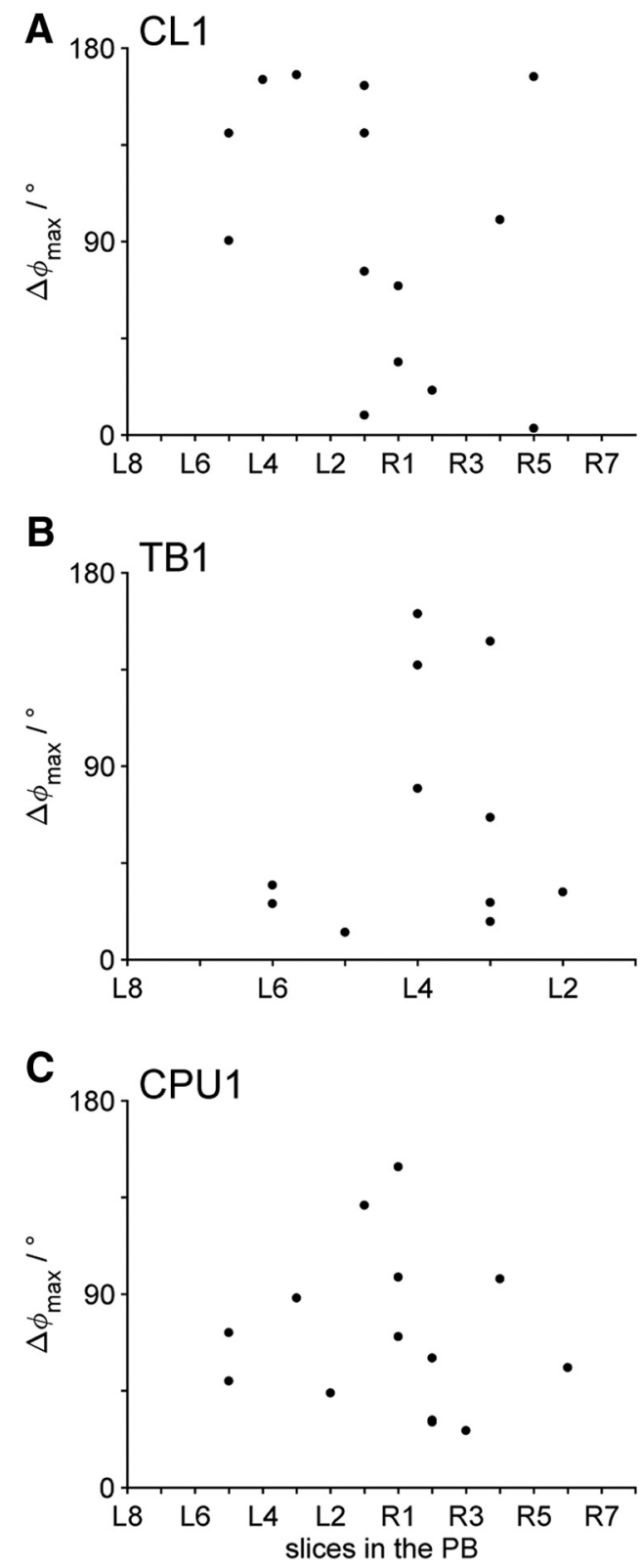

Figure 4. Distances between preferred $E$-vectors and azimuth angles. $\boldsymbol{A}-\boldsymbol{C}$, Distances between preferred $E$-vector angles and preferred azimuth angles (black markers) plotted against the slice of arborization in the PB. Distances were calculated by subtracting the preferred $E$-vector angle from the preferred azimuth of the green spot. Data points are means from two or four stimulus presentations. $\boldsymbol{A}$, Distances of $\mathrm{CL} 1$ neurons $(n=14)$. $\boldsymbol{B}$, Distances of TB1 neurons $(n=11)$. C, Distances of CPU1 neurons $(n=14)$.

ence for the $E$-vector, as shown for a CL1 and a TB1 neuron (Fig. $5 B, C)$, or for the azimuth of the green spot, as shown for a CPU1 and a CPU2 neuron (Fig. 5D,E). Some neurons showed responses to combined stimulation, in which the contributions of $E$-vector tuning and light spot tuning were similar (CL1; Fig. 5F). Nonetheless, in neurons not showing a strong preference, the response to combined stimulation always revealed one higher peak or one deeper trough in spike rate modulation across $360^{\circ}$.

\section{Influence of elevation on azimuth tuning}

In addition to its horizontal component (azimuth), the position of the sun has a vertical component (elevation). Although only 

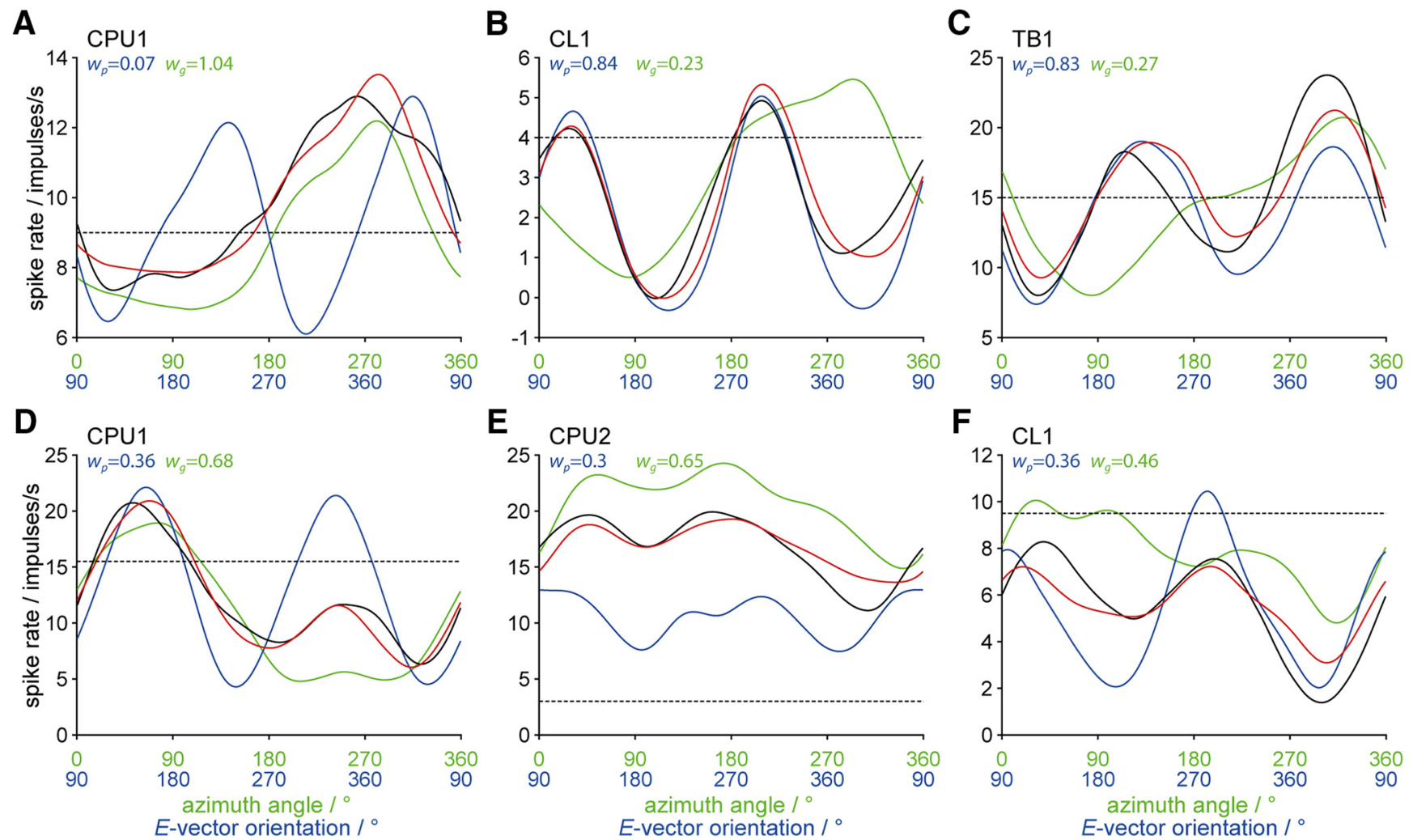

Figure 5. Responses to combined stimulation with polarized light and an unpolarized light spot. $\boldsymbol{A}-\boldsymbol{F}$, Tunings of individual $(X$ neurons to the $E$-vector (blue), to the azimuth of the unpolarized light spot (green), and to both stimuli presented simultaneously (black). Tunings are presented as smoothed stimulus-response curves based on means from four stimulus presentations except for the $E$-vector data in $\mathbf{C}$ and $\boldsymbol{F}$, which are means from two stimulus presentations. During combined stimulation, the $E$-vector was adjusted at $90^{\circ}$ angular distance from the green light spot. Therefore, the $x$-axis shows the orientation of the $E$-vector (blue) separated from the azimuth angle (green). Responses to the $E$-vector alone are shifted by $90^{\circ}$. Solid red lines show the best fit curves resulting from summation of $E$-vector tuning and light spot tuning multiplied by a weighting factor $w_{\mathrm{p}}$ for the $E$-vector tuning and $w_{\mathrm{g}}$ for the azimuth tuning. Dotted lines indicate background activity. $\boldsymbol{A}$, Responses of a CPU1 neuron showing strong preference for the green light spot. $E$-vector tuning: $\Phi_{\max }=40^{\circ} ; p=6 \cdot 10^{-5}$. Azimuth tuning: $\Phi_{\max }=264^{\circ} ; p=2 \cdot 10^{-5}$. Combined response bidirectional tuning: $p=0.83$. Combined response unidirectional tuning: $\Phi_{\max }=249^{\circ} ; p=1 \cdot 10^{-3} \cdot \boldsymbol{B}-\boldsymbol{F}$, Responses of CL1 neurons $(\boldsymbol{B}, \boldsymbol{F}), \mathrm{a}$ TB1 neuron $(\boldsymbol{C})$, a CPU1 neuron $(\boldsymbol{D})$, and a $C P U 2$ neuron (E) to combined stimulation with less pronounced preference for the $E$-vector or the azimuth of the green spot. $\boldsymbol{B}$, Responses of a $\mathrm{CL} 1$ neuron showing a preference for polarized light. $E$-vector tuning: $\Phi_{\max }=120^{\circ} ; p=3 \cdot 10^{-6}$. Azimuth tuning: $\Phi_{\max }=261^{\circ} ; p=2 \cdot 10^{-6}$. Combined response bidirectional tuning: $\Phi_{\max }=117^{\circ} ; p=2 \cdot 10^{-5}$. Combined response unidirectional tuning: $p=$ 0.64. C, Responses of a TB1 neuron. E-vector tuning: $\Phi_{\max }=39^{\circ} ; p=2 \cdot 10^{-5}$. Azimuth tuning: $\Phi_{\max }=285^{\circ} ; p=4 \cdot 10^{-5}$. Combined response bidirectional tuning: $\Phi_{\max }=36^{\circ} ; p=2 \cdot 10^{-4}$. Combined response unidirectional tuning: $p=0.2$. D, Responses of a CPU1 neuron. E-vector tuning: $\Phi_{\max }=152^{\circ} ; p=2 \cdot 10^{-7}$. Azimuth tuning: $\Phi_{\max }=69^{\circ} ; p=8 \cdot 10^{-7}$. Combined response bidirectional tuning: $\Phi_{\max }=154^{\circ} ; p=3 \cdot 10^{-3}$. Combined response unidirectional tuning: $\Phi_{\max }=73^{\circ} ; p=2 \cdot 10^{-3}$. E, Responses of a CPU2 neuron. $E$-vector tuning: $\Phi_{\max }=99^{\circ} ; p=4 \cdot 10^{-3}$. Azimuth tuning: $\Phi_{\max }=145^{\circ} ; p=2 \cdot 10^{-4}$. Combined response bidirectional tuning: $\Phi_{\max }=116^{\circ} ; p=1 \cdot 10^{-2}$. Combined response unidirectional tuning: $\Phi_{\max }=121^{\circ} ; p=1 \cdot 10^{-3} \cdot \boldsymbol{F}_{\text {, }}$ Responses of a CL1 neuron. E-vector tuning: $\Phi_{\max }=104^{\circ} ; p=2 \cdot 10^{-5}$. Azimuth tuning: $\Phi_{\max }=92^{\circ} ; p=5 \cdot 10^{-3}$. Combined response bidirectional tuning: $\Phi_{\max }=119^{\circ} ; p=1 \cdot 10^{-3}$. Combined response unidirectional tuning: $\Phi_{\max }=117^{\circ} ; p=3 \cdot 10^{-3}$.

the azimuth provides compass information, coding for solar elevation might provide daytime-dependent information and thus could aid in time-compensated sun compass navigation. We therefore tested different elevations of the unpolarized green light spot in recordings of four CL1, four TB1, and six CPU1 neurons. Only a few recordings showed an impact of elevation on the azimuth tuning. One CL1 neuron showed a second peak in azimuth tuning when stimulated at low elevations (Fig. 6A). At higher elevations of $50^{\circ}$ or $60^{\circ}$, the second peak disappeared so that the tuning was more directed toward $\Phi_{\max }$. Another phenomenon occurred in a CPU1 neuron: the tuning curve was flat at high elevations and of higher amplitude at low elevations (Fig. $6 B$ ). Of all recordings, these two neurons showed the strongest influence of elevation on azimuth tuning. The general effects on the correlation strength of the tunings were small (Fig. 6C). Different elevations changed the significance of the correlation coefficient only in one CL1 (red), one TB1 (blue), and one CPU1 neuron (purple). All other neurons were either responsive to all tested elevations $(n=10)$ or to none of them $(n=1)$. Elevations different from $45^{\circ}$ were often tested later in the recording so that tuning parameters might have been altered by a change of neuronal background state. Across all cell types, the correlation strength was most dispersed between elevations, whereas tuning amplitude and width were only slightly affected. Nonetheless, no common systematic change of tuning parameters occurred in any neuron type.

\section{Discussion}

Side-specific azimuth representation in tangential inputs to the CBL

In this study, we analyzed the relevance of solar azimuth and elevation and of the zenithal $E$-vector for the internal representation of heading direction in locust CX neuropils. TL2 and TL3 neurons are the likely input elements of polarization and azimuth information to the CX (Pegel et al., 2018). We identified two TL2 subtypes arborizing in different parts of the lateral bulb and different layers of the CBL (Müller et al., 1997) preferring a bright light spot on either the ipsilateral (TL2a) or contralateral side (TL2b) of the animal. Our findings are similar to characteristics of ring neurons ( $\mathrm{R}$ neurons; equivalent to locust TL neurons) of 
A

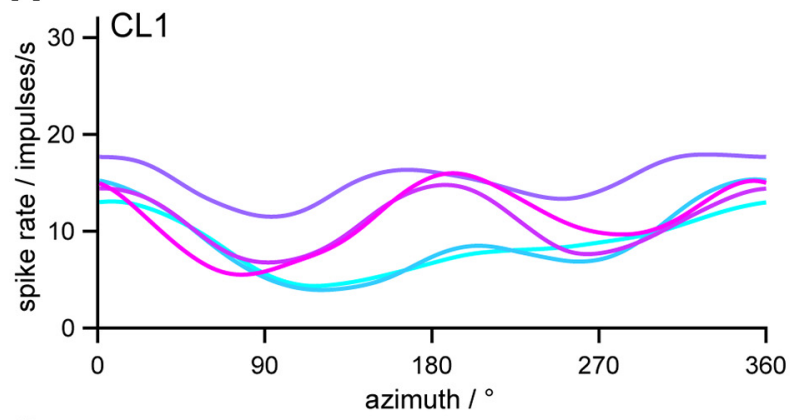

C

CL1

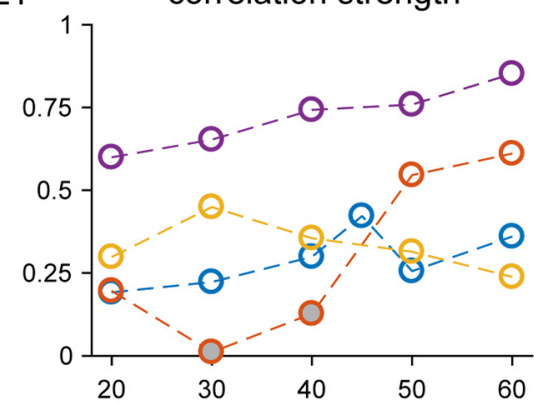

B

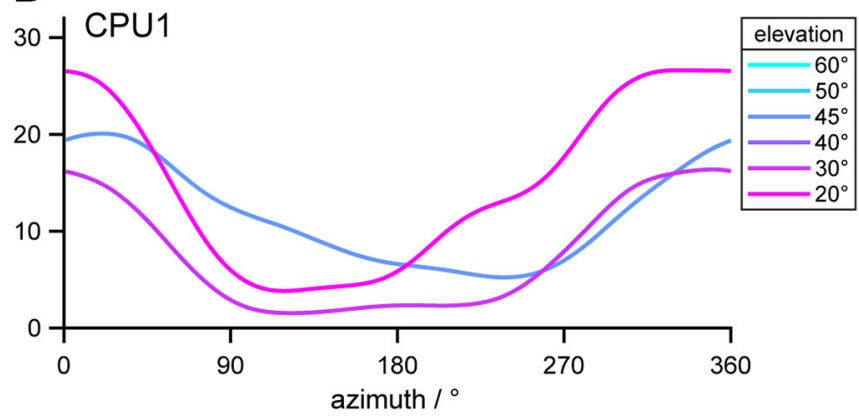

tuning amplitude
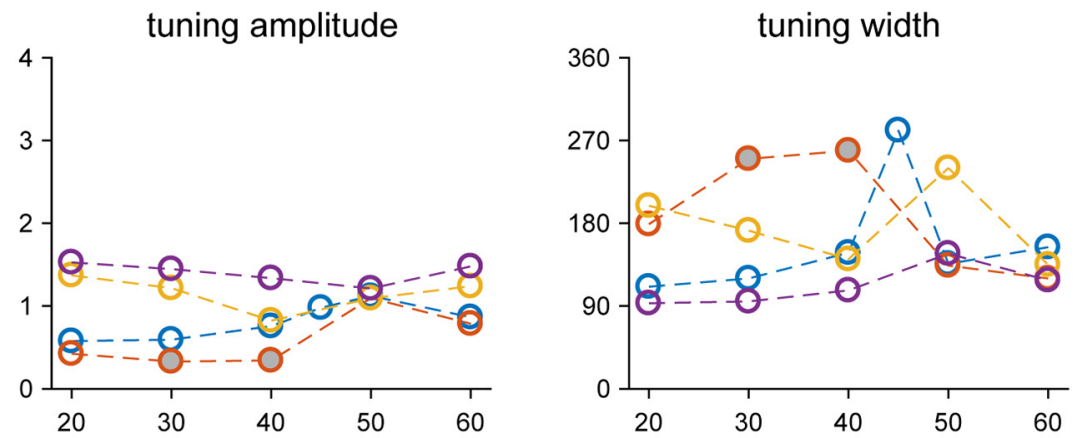

TB1
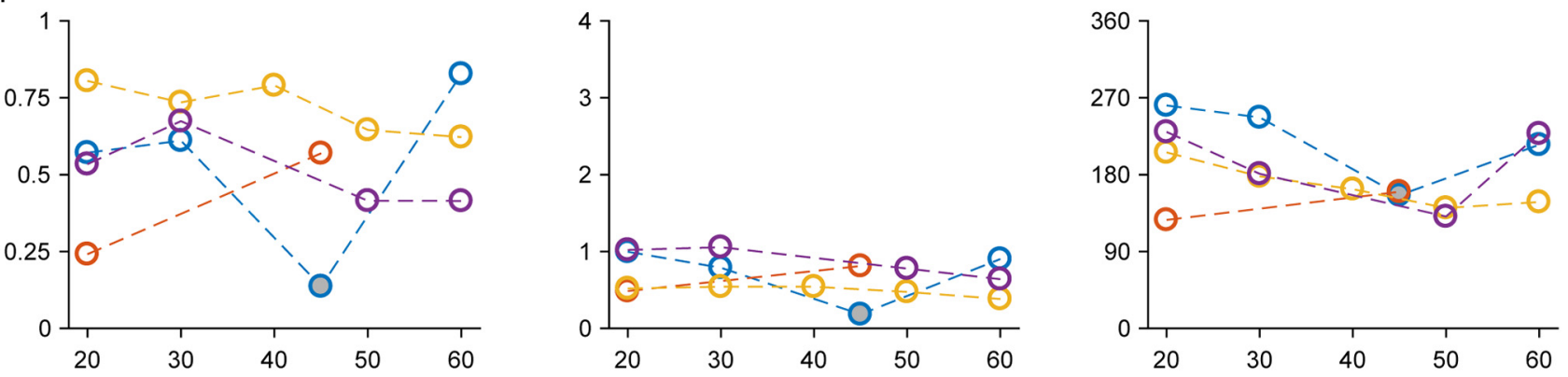

CPU1
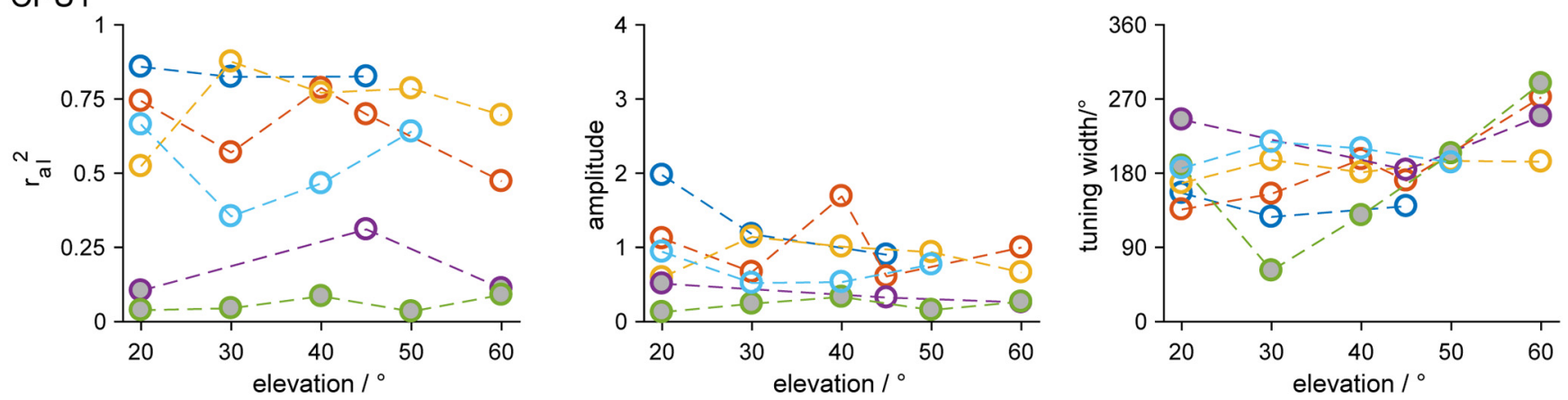

Figure 6. Influence of stimulus elevation on tuning parameters of $C L 1, T B 1$, and CPU1 neurons. $A, B$, Smoothed stimulus-response curves of a $C L 1$ neuron $(\boldsymbol{A})$ and a $C P U 1$ neuron $(B)$ to rotation of the green light spot presented at different elevations. Number of trials: $n=4$ for all elevations in the CL1 neuron and the $45^{\circ}$ elevation in the CPU1 neuron and $n=6$ for all other elevations in the CPU1 neuron. C, Tuning parameters of all recorded CL1, TB1, and CPU1 neurons. Tuning amplitude, tuning width, and correlation strength $\left(r_{\text {al }}{ }^{2}\right)$ of the average stimulus-response curve of each neuron plotted against the tested elevation. Colors code for individual neurons of the respective cell type. Open circles indicate data from significant responses; circles filled in gray indicate data from nonsignificant responses. Data from the CL1 neuron coded in red are from the same neuron as data in $\boldsymbol{A}$ and data from the CPU1 neuron coded in dark blue are from the same neuron as data in $\boldsymbol{B}$. Number of trials ranged from $n=2$ to $n=6$ in all cell types.

Drosophila. R neurons arborizing in ventral parts of the bulb have contralateral receptive fields for visual cues and innervate outer layers of the ellipsoid body, whereas $\mathrm{R}$ neurons connecting microglomeruli in dorsal parts of the bulb to inner layers of the ellipsoid body respond to ipsilateral targets (Shiozaki and Ka- zama, 2017). The latter also encode the recent visual experience of targets. These data suggest that different layers of the CBL/ellipsoid body not only receive input from different parts of the visual field, but also different types of information related to memory and decision making. 


\section{Compass representations in CX slices}

A compass-like representation of zenithal $E$-vectors in the $\mathrm{PB}$ has been shown for TB1, CPU1, and CP1/CP2 neurons (Heinze and Homberg, 2007). These findings are confirmed here for TB1 and CPU1 neurons. As in Heinze and Homberg (2007), all regression lines had a positive slope and, in CPU1 neurons, covered $\sim 360^{\circ}$ of compass directions from L8-R8. For TB1 neurons, the slope of the regression line representing $E$-vectors differed from that shown by Heinze and Homberg (2007), but this may be because of the low sample size for TB1 neurons studied here. We found no correlation between preferred $E$-vector and PB slice in CL1 neurons, confirming the findings of Heinze and Homberg (2009).

Although Heinze and Homberg (2007) calculated coefficients using a linear-linear correlation analysis, we used a circular-linear approach, which affects the results in a negative way as follows: (1) with small sample size $(n<10)$, the correlation has only low reliability (Kempter et al., 2012) and our sample size of TB1 neurons was just above this number and (2) for the analysis of axial data such as the preferred $E$-vectors, the angles need to be doubled to convert them into a circular variable. This artificially increases the dispersion of data, thus decreasing the likelihood of correlation or, in the case of correlation, its strength. Nonetheless, the circular-linear analysis is highly superior to a linearlinear model. For the analysis of circularity, data points are already present multiple times on the $y$-axis. This eliminates the need to select the data points within the circular space to be used for the linear-linear correlation analysis, which strongly influences its result.

In addition to $E$-vector topography, we found a topographical representation of azimuth (green light spot) in CL1 and CPU1 neurons. In both cell types, the regression line had a negative slope from L8 to R8, opposite to that for $E$-vector coding. It covered a range of $638^{\circ}$ in CL1 neurons. This range is close to the twofold representation of $360^{\circ}$ space of E-PG neurons (equivalents to locust CL1 neurons) in the fly (Green et al., 2017), which was also a necessary assumption for a computational model of path integration in bees (Stone et al., 2017). In contrast, the CPU1 columnar output neurons represented only $464^{\circ}$ of azimuthal directions. Because CPU1 neurons have so far not been found in the two outermost columns (R7/8, L7/8) and also appear to be absent in these columns in bees (Stanley Heinze, personal communication), this range might be further reduced to $340^{\circ}$ from R6-L6. Being the output elements of the CX, CPU1 neurons, in contrast to CL1 neurons, may not show a purely sensory signal anymore, but rather a steering motor command signal. It is therefore reasonable that columnar outputs of the $\mathrm{PB}$ represent a range of just $180^{\circ}$ in one $\mathrm{PB}$ hemisphere because neurons from one hemisphere have their presynaptic arborizations in the same lateral accessory lobe so that they might elicit turning behavior either to the left or to the right.

\section{Elevation independence}

In Drosophila, $\mathrm{R}$ neurons have approximately circular receptive fields for a bright bar defined by a preferred azimuth and preferred elevation (Seelig and Jayaraman, 2013). In contrast, in the locust, elevation of the green light spot was not encoded by PB neurons because most neurons were either responsive to all elevations or to none. Whether this difference is species specific or results from the fact that we did not collect data from TL neurons, only from downstream columnar neurons that have not been studied in Drosophila, remains to be established. The elevation independence in the tuning to the green light suggests that only the azimuth information is relevant for the coding properties of the neurons studied here. Theoretically, information on solar elevation could be useful for daytime estimation, but is apparently ignored in birds (Keeton, 1974; Wiltschko, 1980), honey bees (von Frisch, 1965), and desert ants (Duelli and Wehner, 1973) in favor of a circadian clock input for time-compensated sun compass orientation.

\section{Biological significance}

Honeybees, desert ants, and dung beetles are able to use both the sun and the polarization pattern of the sky as navigational cues (Brines and Gould, 1979; Wehner and Müller, 2006; el Jundi et al., 2015). Both signals are processed in the CX. In addition to encoding the plane of zenithal polarized light, CX neurons of dung beetles (el Jundi et al., 2015) and monarch butterflies (Heinze and Reppert, 2011) show azimuth-dependent responses to a bright light spot assumed to represent the sun. In Drosophila, azimuthal tunings to bright light spots are topographically represented in the ellipsoid body and PB (Seelig and Jayaraman, 2015; Turner-Evans et al., 2017). We show here that both the representation of zenithal $E$-vectors and the azimuth of an unpolarized light spot are mapped to the PB slices in a compass-like manner.

The two internal compasses for $E$-vector orientation and azimuth angle, however, do not support each other; they differ, not only in their orientation, but also in the total angular range they represent across the $\mathrm{PB}$. Moreover, there is no $90^{\circ}$ distance between preferred $E$-vector and preferred azimuth in any of the $\mathrm{PB}$ slices, so that the natural distance between zenithal $E$-vector and sun is not encoded. This raises the question of how the two compasses may interact in a natural setting. All data presented here were obtained from animals harnessed tightly for intracellular recordings. It is conceivable that the measured offset of the two compasses may be altered by active locomotion and turning movements of the locust. As shown in cockroaches (Varga and Ritzmann, 2016) and flies (Turner-Evans et al., 2017), certain CX neurons are tuned, not only to heading direction, but also to turning velocity of the animal and even to turning history. Addressing these effects in locusts would likewise require recordings during turning movements, ideally in freely moving animals.

Confinement of polarized light stimulation to a small area in the zenith has probably also affected the E-vector representation in the CX. Bees and ants need to see a sufficiently large part of the sky polarization pattern to calculate solar position and make systematic errors when they observe only a small part of the blue sky (Rossel and Wehner, 1984; Rossel, 1993; Wehner and Müller, 2006). Bech et al. (2014) showed that locust CX neurons encode sky-like patterns of differently oriented $E$-vectors. If the receptive fields of CX neurons are not zenith centered, then the internal $E$-vector compass based on the zenithal E-vector may substantially differ from a polarization compass based on a complete Rayleigh sky. Support for this hypothesis comes from a computational model of an insect-inspired polarization compass (Gkanias et al., 2018). Signals from a fan-shaped arrangement of $E$-vector analyzers as present in the dorsal rim area of the eye were fed into an array of compass neurons covering a $360^{\circ}$ azimuth range. When stimulating the $E$-vector analyzers with a rotating polarizer instead of a Rayleigh pattern of $E$-vectors, the array of compass neurons showed an $\sim 180^{\circ}$-representation of directions instead of $360^{\circ}$ and, moreover, a paradox mirror-symmetric topography compared with Rayleigh sky stimulation. Unfortunately, stimulation with a pattern of polarizers mimicking the Rayleigh sky is hardly possible in a laboratory setting. Therefore, the polarization compass in the CX based on matched-filter 
mechanisms for the Rayleigh sky may indeed be organized differently than revealed by the "single-polarizer" stimulation used here.

In conclusion, our results support the assumption that CX neurons are involved in navigation, not only by using a sky polarization compass, but also an azimuth compass possibly representing the sun. Both internal compasses emerge in neurons of the $\mathrm{PB}$ and are present in columnar output neurons. Experiments under the open sky are likely to reveal how both compasses interact with each other in a natural setting to produce a robust head direction signal.

\section{References}

Batschelet E (1981) Circular statistics in biology. London: Academic.

Bech M, Homberg U, Pfeiffer K (2014) Receptive fields of locust brain neurons are matched to polarization patterns of the sky. Curr Biol 24:2124-2129.

Bockhorst T, Homberg U (2015) Amplitude and dynamics of polarizationplane signaling in the central complex of the locust brain. J Neurophysiol 113:3291-3311.

Brandt R, Rohlfing T, Rybak J, Krofczik S, Maye A, Westerhoff M, Hege HC, Menzel R (2005) Three-dimensional average-shape atlas of the honeybee brain and its applications. J Comp Neurol 492:1-19.

Brines ML, Gould JL (1979) Bees have rules. Science 206:571-573.

Brunner D, Labhart T (1987) Behavioural evidence for polarization vision in crickets. Physiological Entomology 12:1-10.

Clements AN, May TE (1974) Studies on locust neuromuscular physiology in relation to glutamic acid. J Exp Biol 60:673-705.

Collett TS (1992) Landmark learning and guidance in insects. Philos Trans R Soc Lond B 337:295-303.

Dacke M, Nordström P, Scholtz CH (2003) Twilight orientation to polarized light in the crepuscular dung beetle Scarabaeus zambesianus. J Exp Biol 206:1535-1543.

Duelli P, Wehner R (1973) The spectral sensitivity of polarized light orientation in Cataglyphis bicolor (Formicidae, Hymenoptera). J Comp Physiol $86: 37-53$.

el Jundi B, Pfeiffer K, Heinze S, Homberg U (2014a) Integration of polarization and chromatic cues in the insect sky compass. J Comp Physiol A Neuroethol Sens Neural Behav Physiol 200:575-589.

el Jundi B, Smolka J, Baird E, Byrne MJ, Dacke M (2014b) Diurnal dung beetles use the intensity gradient and the polarization pattern of the sky for orientation. J Exp Biol 217:2422-2429.

el Jundi B, Warrant EJ, Byrne MJ, Khaldy L, Baird E, Smolka J, Dacke M (2015) Neural coding underlying the cue preference for celestial orientation. Proc Natl Acad Sci U S A 112:11395-11400.

Frost BJ, Mouritsen H (2006) The neural mechanisms of long distance animal navigation. Curr Opin Neurobiol 16:481-488.

Gkanias E, Risse B, Mangan M, Webb B (2018) From skylight input to behavioural output: a computational model of the insect polarised light compass. Available at https://www.biorxiv.org/content/10.1101/ $504597 \mathrm{vl}$.

Gould JL (1998) Sensory bases of navigation. Curr Biol 8:R731-R738.

Green J, Adachi A, Shah KK, Hirokawa JD, Magani PS, Maimon G (2017) A neural circuit architecture for angular integration in Drosophila. Nature 546:101-106.

Heinze S (2014) Polarized-light processing in insect brains: recent insights from the desert locust, the monarch butterfly, the cricket, and the fruit fly. In: Polarized light and polarization vision in animal sciences (Horváth G, ed), pp 61-111. Berlin, Heidelberg: Springer.

Heinze S, Homberg U (2007) Maplike representation of celestial E-vector orientations in the brain of an insect. Science 315:995-997.

Heinze S, Homberg U (2008) Neuroarchitecture of the central complex of the desert locust: intrinsic and columnar neurons. J Comp Neurol 511: $454-478$.

Heinze S, Homberg U (2009) Linking the input to the output: new sets of neurons complement the polarization network in the locust central complex. J Neurosci 29:4911-4921.

Heinze S, Reppert SM (2011) Sun compass integration of skylight cues in migratory monarch butterflies. Neuron 69:345-358.

Held M, Berz A, Hensgen R, Muenz TS, Scholl C, Rössler W, Homberg U, Pfeiffer K (2016) Microglomerular synaptic complexes in the sky- compass network of the honeybee connect parallel pathways from the anterior optic tubercle to the central complex. Front Behav Neurosci 10:186.

Homberg U (2015) Sky compass orientation in desert locusts - evidence from field and laboratory studies. Front Behav Neurosci 9:346.

Homberg U, Heinze S, Pfeiffer K, Kinoshita M, el Jundi B (2011) Central neural coding of sky polarization in insects. Philos Trans R Soc Lond B Biol Sci 366:680-687.

Keeton WT (1974) The orientational and navigational basis of homing in birds. In: Recent advances in the study of behavior (Lehrman DS, Hinde RA, Shaw E, eds), pp 47-132. New York: Academic.

Kempter R, Leibold C, Buzsáki G, Diba K, Schmidt R (2012) Quantifying circular-linear associations: hippocampal phase precession. J Neurosci Methods 207:113-124

Klagges BR, Heimbeck G, Godenschwege TA, Hofbauer A, Pflugfelder GO, Reifegerste R, Reisch D, Schaupp M, Buchner S, Buchner E (1996) Invertebrate synapsins: a single gene codes for several isoforms in Drosophila. J Neurosci 16:3154-3165.

Kurylas AE, Rohlfing T, Krofczik S, Jenett A, Homberg U (2008) Standardized atlas of the brain of the desert locust, Schistocerca gregaria. Cell Tissue Res 333:125-145.

Labhart T, Meyer EP (1999) Detectors for polarized skylight in insects: a survey of ommatidial specializations in the dorsal rim area of the compound eye. Microsc Res Tech 47:368-379.

Mappes M, Homberg U (2004) Behavioral analysis of polarization vision in tethered flying locusts. J Comp Physiol A Neuroethol Sens Neural Behav Physiol 190:61-68.

Menzel R, Greggers U, Smith A, Berger S, Brandt R, Brunke S, Bundrock G, Hülse S, Plümpe T, Schaupp F, Schüttler E, Stach S, Stindt J, Stollhoff N, Watzl S (2005) Honey bees navigate according to a map-like spatial memory. Proc Natl Acad Sci U S A 102:3040-3045.

Merlin C, Heinze S, Reppert SM (2012) Unraveling navigational strategies in migratory insects. Curr Opin Neurobiol 22:353-361.

Müller M, Homberg U, Kühn A (1997) Neuroarchitecture of the lower division of the central body in the brain of the locust (Schistocerca gregaria). Cell Tissue Res 288:159-176.

Ofstad TA, Zuker CS, Reiser MB (2011) Visual place learning in Drosophila melanogaster. Nature 474:204-207.

Pegel U, Pfeiffer K, Homberg U (2018) Integration of celestial compass cues in the central complex of the locust brain. J Exp Biol 221:jeb171207.

Pfeiffer K, Kinoshita M, Homberg U (2005) Polarization-sensitive and light-sensitive neurons in two parallel pathways passing through the anterior optic tubercle in the locust brain. J Neurophysiol 94:3903-3915.

Reppert SM, Guerra PA, Merlin C (2016) Neurobiology of monarch butterfly migration. Annu Rev Entomol 61:25-42.

Rossel S (1993) Navigation by bees using polarized skylight. Comp Biochem Physiol A 104:695-708.

Rossel S, Wehner R (1984) How bees analyse the polarization patterns in the sky: experiments and model. J Comp Physiol A Neuroethol Sens Neural Behav Physiol 155:605-613.

Schmeling F, Wakakuwa M, Tegtmeier J, Kinoshita M, Bockhorst T, Arikawa K, Homberg U (2014) Opsin expression, physiological characterization and identification of photoreceptor cells in the dorsal rim area and main retina of the desert locust, Schistocerca gregaria. J Exp Biol 217:3557-3568.

Seelig JD, Jayaraman V (2013) Feature detection and orientation tuning in the Drosophila central complex. Nature 503:262-266.

Seelig JD, Jayaraman V (2015) Neural dynamics for landmark orientation and angular path integration. Nature 521:186-191.

Shiozaki HM, Kazama H (2017) Parallel encoding of recent visual experience and self-motion during navigation in Drosophila. Nat Neurosci 20: $1395-1403$.

Stone T, Webb B, Adden A, Weddig NB, Honkanen A, Templin R, Wcislo W, Scimeca L, Warrant E, Heinze S (2017) An anatomically constrained model for path integration in the bee brain. Curr Biol 27:3069-3085.e11.

Träger U, Wagner R, Bausenwein B, Homberg U (2008) A novel type of microglomerular synaptic complex in the polarization vision pathway of the locust brain. J Comp Neurol 506:288-300.

Turner-Evans D, Wegener S, Rouault H, Franconville R, Wolff T, Seelig JD, Druckmann S, Jayaraman V (2017) Angular velocity integration in a fly heading circuit. Elife 6:e23496.

Varga AG, Ritzmann RE (2016) Cellular basis of head direction and contextual cues in the insect brain. Curr Biol 26:1816-1828. 
Varga AG, Kathman ND, Martin JP, Guo P, Ritzmann RE (2017) Spatial navigation and the central complex: sensory acquisition, orientation, and motor control. Front Behav Neurosci 11:4.

von Frisch K (1949) Die Polarisation des Himmelslichtes als orientierender Faktor bei den Tänzen der Bienen. Experientia 5:142-148.

von Frisch K (1965) Tanzsprache und Orientierung der Bienen. Berlin/ Heidelberg/New York: Springer.

Wehner R (1984) Astronavigation in insects. Annu Rev Entomol 29: 277-298.

Wehner R, Müller M (2006) The significance of direct sunlight and polarized skylight in the ant's celestial system of navigation. Proc Natl Acad Sci U S A 103:12575-12579.
Weir PT, Dickinson MH (2012) Flying Drosophila orient to sky polarization. Curr Biol 22:21-27.

Wiltschko R (1980) Die Sonnenorientierung der Vögel. I. Die Rolle der Sonne im Orientierungssystem und die Funktionsweise des Sonnenkompasses. J Ornithol 121:121-143.

Wystrach A, Graham P (2012) View-based matching can be more than image matching: the importance of considering an animal's perspective. Iperception 3:547-549.

Zar JH (1999) Biostatistical analysis, Ed 4. Upper Saddle River, NJ: Prentice Hall.

Zars T (2009) Spatial orientation in Drosophila. J Neurogenet 23:104110. 